\title{
Quantidade de Treino e Reorganização de Classes de Equivalência com Crianças
}

\author{
André Lepesqueur Cardoso* (D) \& Raquel Maria de Melo \\ Universidade de Brasília, Brasília, DF, Brasil
}

\begin{abstract}
RESUMO - Investigou-se o efeito da revisão da linha de base na formação, reorganização e restabelecimento de classes de equivalência. Quatro crianças (5-6 anos) realizaram duas condições (Com e Sem Revisão das relações treinadas antes dos testes), com variação da ordem de exposição. Na Fase 1, foram treinadas as relações de linha de base (AC, BC e AD) e testadas as que evidenciam classes de equivalência. Na Fase 2, apenas a relação AD foi revertida e realizados os testes de reorganização. Na Fase 3, os treinos e testes da Fase 1 foram repetidos. Verificou-se que para três participantes, apenas da Condição Com Revisão, que as classes foram reorganizadas, conforme o treino de reversão, se restabeleceram com o retorno à linha de base.
\end{abstract}

PALAVRAS-CHAVE: equivalência, reorganização de classes, revisão de linha de base, reversão das relações de linha de base, crianças

\section{Training Quantity and Reorganization of Equivalence Classes with Children}

\begin{abstract}
The effects of baseline review trials were investigated in the formation, reorganization and reestablishment of equivalence classes. Four children (5-6 years old) were exposed to two conditions (With Review and Without Review of the trained relations before the tests) in different orders. In Phase 1, baseline relations (AB, BC and AD) were trained and relations that showed evidence of equivalence were tested. In Phase 2, only relation AD was reversed and reorganization tests were conducted. In Phase 3, the training and tests conducted in Phase 1 were repeated. For three participants, only in the With Review condition, classes were reorganized, as expected from the reversal training, and were reestablished upon return to baseline.
\end{abstract}

KEYWORDS: equivalence, classes reorganization, baseline review, reversal of baseline relation, children

Equivalência de estímulos é um tema relevante na Análise do Comportamento, uma vez que é uma alternativa para a compreensão de fenômenos envolvidos na aprendizagem de comportamentos simbólicos, tais como leitura alfabética e musical, relações numéricas e conceitos, e que estão relacionados com a aquisição de desempenhos novos ou emergentes (de Rose, de Souza, \& Hanna, 1996; Lynch \& Cuvo, 1995; Prado \& de Rose, 1999; Sidman, 1994; Tena \& Velázquez, 1997). São também citadas na literatura implicações para a compreensão de comportamentos adquiridos no contexto social, como os que estão relacionadas com estereótipos e autoconceito (Barnes, Lawlor, Smeets, \& Roche, 1996; Carvalho \& de
Rose, 2014; Dymond \& Barnes, 1994; Watt, Keenan, Barnes, \& Cairns, 1991).

Os estímulos são denominados de equivalentes quando se tornam substituíveis em sua função de controle comportamental. Isto é, quando as funções adquiridas por um estímulo são transferidas para os demais estímulos da classe (Albuquerque \& Melo, 2005; Sidman, 1994). Os estudos sobre equivalência consistem, em geral, de uma etapa de treino de discriminações condicionais e uma etapa de teste para verificar a emergência de novas relações condicionais. Discriminações condicionais podem ser ensinadas pelo procedimento de pareamento ao modelo (do inglês, matching to sample). Neste procedimento, diante

*E-mail: andrelecardoso@gmail.com

- Submetido: 04/09/2016; Revisado: 25/10/2016; Aceito: 16/02/2018. 
de cada estímulo modelo somente a seleção do estímulo de comparação correspondente ao modelo é reforçada, enquanto a seleção de uma das outras comparações não resultará em reforço. A verificação de relações emergentes, não diretamente ensinadas, é realizada a partir dos testes de reflexividade, simetria e transitividade (Sidman \& Tailby, 1982). A demonstração de tais desempenhos indica que foram formadas classes de estímulos equivalentes.

Quando novas relações condicionais são ensinadas, e estas são incompatíveis com as classes anteriormente formadas (conflitantes), as classes podem permanecer inalteradas. Nesse caso, os participantes não respondem de acordo com o que foi mais recentemente ensinado, o que indica que as classes não foram modificadas ou reorganizadas (e.g., Pilgrim, Chambers, \& Galizio, 1995; Pilgrim \& Galizio, 1990; 1995; Saunders, Saunders, Kirby, \& Spradlin, 1988). Estudos sobre reorganização de classes de equivalência destinam-se, portanto, a verificar se novas classes são formadas a partir do ensino de relações condicionais diferentes, ou incompatíveis com as classes de equivalência previamente formadas.

$\mathrm{O}$ procedimento mais comum para investigar a reorganização de classes é a reversão. Inicialmente, são treinadas as discriminações condicionais de linha de base (e.g., $\mathrm{A} 1 \mathrm{~B} 1, \mathrm{~A} 2 \mathrm{~B} 2$ e $\mathrm{B} 1 \mathrm{C} 1, \mathrm{~B} 2 \mathrm{C} 2$ ) e realizados os testes de formação de classes de equivalência (e.g., A1B1C1, A2B2C2). Posteriormente, são revertidas as relações condicionais entre os elementos de, pelo menos, dois conjuntos de estímulos (e.g., B1C2 e B2C1), sendo mantidas as demais relações condicionais previamente treinadas (e.g., A1B1 e A2B2). Caso o desempenho do participante seja preciso nestes testes, ou seja, coerente com as novas relações condicionais treinadas, esta seria uma evidência da reorganização das classes de equivalência anteriormente estabelecidas (e.g., classes reorganizadas: Classe 1 A1B1C2 e Classe 2-A2B2C1).

Um dos primeiros estudos que demonstrou a reorganização de classes, com o procedimento de reversão, foi realizado por Dube, McIlvane, Mackay e Stoddard (1987). Esse estudo tinha como objetivo avaliar o efeito de dois tipos diferentes de reforçadores (reforço específico) na formação (Experimento 1), expansão (Experimento 2) e reorganização de classes (Experimento 3) com dois adultos com atraso no desenvolvimento. Os resultados demonstraram a formação, reversão das relações condicionais, expansão e reorganização das classes previamente estabelecidas. De acordo com os autores, as contingências de reforçamento mais recentes (reversão de algumas relações condicionais) foram suficientes para sobrepor a história de treino inicial (ou linha de base original).

Pilgrim e Galizio $(1990,1995)$ investigaram, com estudantes universitários, o efeito da reversão de relações condicionais de linha de base nas classes de equivalência e, em contraste com os resultados de Dube et al. (1987), foi verificada estabilidade e resistência à mudança das classes originalmente estabelecidas. Desempenhos consistentes com a linha de base revertida ocorreram apenas nos testes de simetria. Com crianças de 5-7 anos e tarefas de pareamento ao modelo com objetos concretos, Pilgrim et al. (1995) verificaram dificuldade na etapa de treino de novas relações condicionais com o procedimento de reversão. Nos testes, foi observada a ocorrência de erros e os desempenhos não evidenciaram a reorganização das classes de equivalência. No entanto, Goyos (2000) verificou que crianças de 4 a 5 anos somente demonstraram reorganização das classes de equivalência após o ensino de um nome comum para os elementos de cada classe. Diferentemente, Saunders, Drake e Spradlin (1999) verificaram a reorganização de classes de equivalência com crianças de 4-5 anos após a reversão de relações condicionais, mas sem a necessidade de ensinar um nome comum para os membros das classes. Estas divergências entre os resultados de diferentes estudos indicam a necessidade de investigar variáveis que afetam as respostas dos participantes nos testes de reorganização de classes e a manutenção de desempenhos coerentes com as contingências de treino original ou com o treino de reversão.

Entretanto, outros estudos descritos na literatura demonstram que é possível alterar as classes de equivalência previamente formadas (Almeida \& Haydu, 2009; Folsta \& de Rose, 2007; Garotti \& de Rose, 2007). Garotti e de Rose (2007), por exemplo, investigaram o efeito de revisões de linha de base (tentativas adicionais de treino antes dos testes) na formação e, posterior, reorganização de classes de equivalência. Foram realizados dois experimentos, com estudantes de 15-24 anos. Cada experimento era composto por cinco fases e foram utilizados estímulos visuais sem referentes na língua portuguesa (estímulos arbitrários). $\mathrm{Na}$ Fase 1 foram treinadas as relações condicionais $\mathrm{AB}, \mathrm{BC}$ e $\mathrm{AD}$ e verificada a formação de três classes com quatro elementos cada. Na Fase 2 foram realizados os treinos com reversão das relações $\mathrm{AD}$ de linha de base (ADr), os treinos $\mathrm{AB}$ e $\mathrm{BC}$, e os testes de reorganização das classes. Na Fase 3 foi realizado o treino da relação $\mathrm{AE}$ e repetidos os demais treinos e testes da Fase 2. Na Fase 4 foram realizados os treinos de reversão das relações $\mathrm{BC}$, sendo mantidos os treinos AC e ADr, e os testes de reorganização. Na Fase 5, os participantes foram novamente expostos aos treinos das relações de linha de base original (AC, BC, AD e DE) e aos testes para verificar se as classes inicialmente formadas foram restabelecidas. No Experimento 2, com outros três (3) participantes, foi inserido, em cada fase, um bloco de tentativas das relações treinadas (revisões da linha de base) antes de cada teste (simetria, transitividade e reflexividade). Os testes foram realizados em sonda e em extinção, com tentativas de treino misturadas com tentativas de teste e sem consequências diferenciais para respostas corretas e incorretas.

No Experimento 1, foi verificado que os quatro participantes apresentaram resultados inconsistentes nos testes realizados após os treinos de reversão, sendo que 
o desempenho variava entre coerente com as relações revertidas, com as relações originais de linha de base, ou com nenhuma dessas. Portanto, não foi verificada a reorganização das classes. No Experimento 2, com a inclusão de revisões de linha de base, os resultados mostraram que todos os participantes organizaram e reorganizaram as classes.

No estudo de Garotti e de Rose (2007), portanto, foi observado que a reorganização das classes ocorreu apenas quando tentativas adicionais das relações treinadas (revisões de linha de base) precediam os testes de equivalência. Os autores consideraram que as tentativas de revisão de linha de base funcionaram como um estímulo contextual que indicava a contingência em vigor em cada fase. Entretanto, é provável que a exposição a mais tentativas de treino no Experimento 2 tenha fortalecido as relações aprendidas em cada fase e, portanto, os resultados nos testes podem indicar o efeito da contingência de treino mais recente (em comparação com a contingência mais antiga). O bloco de revisão de linha de base era realizado em extinção e era repetido até que fosse atingido o critério de dois ou menos erros. É possível também que o desempenho nestas tentativas tenha sido mantido por reforçadores não programados explicitamente pelos experimentadores, mas relacionados com a tarefa, como, por exemplo, o encerramento das tentativas de revisão e a mudança para os blocos de teste.

Outro aspecto relevante é a diferença nos resultados dos estudos previamente citados com adultos e crianças. Tais discrepâncias sugerem que experiências prévias dos participantes com relações simbólicas, tais como as envolvidas na linguagem e em conceitos, podem afetar o desempenho nos testes de reorganização de classes de equivalência. Hanna et al. (2008), por exemplo, verificaram desempenhos mais precisos em tarefas que avaliavam a emergência de novas relações condicionais para estudantes de cursos da área de exatas em comparação com estudantes de cursos de humanas. A diferença em relação ao repertório adquirido, antes do estudo, dos estudantes de exatas em disciplinas que ensinam diferentes relações simbólicas como uma matemática, física e química pode ter contribuído para os resultados observados.

Tendo em vista os resultados positivos do estudo de Garotti e de Rose (2007) com adultos, o presente estudo consistiu em uma replicação sistemática, com algumas alterações metodológicas para adequar os procedimentos à faixa etária dos participantes. Para diminuir o efeito da exposição prévia a situações que envolvem treino de relações simbólicas em ambientes não experimentais, participaram do estudo crianças de 5 a 6 anos; foram utilizados estímulos abstratos coloridos; foi realizado apenas um treino de reversão (ADr), o que reduziu o número de estímulos em cada classe (de cinco para quatro) e, consequentemente, a quantidade de testes de reorganização de classes (de dois para um); foram apresentadas consequências diferencias para respostas corretas e incorretas nos blocos de revisão de linha de base; foi utilizado o critério de $89 \%$ de acerto nos testes de formação de classes de equivalência da Fase 1, ao invés de um critério de estabilidade; e foi utilizado o delineamento de sujeito único. Tal delineamento permitiu contrabalancear a ordem de exposição as duas condições experimentais, que se diferenciavam pela presença e ausência de tentativas de revisão de linha de base antes dos testes de formação e reorganização de classes. Desta forma, o estudo permitiu investigar, para os mesmos participantes expostos as duas condições, se a reorganização de classes depende da inclusão das tentativas adicionais de treino de linha de base e se a ordem de exposição as condições (Sem Revisão e Com Revisão) afeta o desempenho nos testes de reorganização de classes.

\section{MÉTODO}

\section{Participantes}

Participaram inicialmente do estudo oito crianças (P1 a P8) com desenvolvimento típico, com idade média de cinco anos e oito meses, não alfabetizadas, e procedentes de uma instituição pública de educação infantil localizada em Brasília. Porém, quatro crianças não finalizam o estudo em decorrência do término do ano letivo. As quatro crianças que completaram o estudo, foram três meninos (P2, P4 e P5) e uma menina (P6).

O estudo foi avaliado e aprovado pelo Comitê de Ética em Pesquisa do Instituto de Ciências Humanas (CEP/ IH) da Universidade de Brasília, em outubro de 2012, com número de registro de 13-07/2012. A participação das crianças foi condicionada à autorização da escola e assinatura dos responsáveis do Termo de Consentimento Livre e Esclarecido, após descrição dos objetivos gerais e das principais características da pesquisa. Vale ressaltar que as crianças foram consultadas sobre o interesse em participar e esclarecidas sobre as atividades desenvolvidas no computador.

\section{Local, Equipamentos e Materiais}

A coleta de dados foi realizada na sala de informática da escola de educação infantil, em um espaço de $10 \mathrm{~m}^{2}$, especialmente reservado para a pesquisa, com uma mesa e duas cadeiras. Brinquedos e jogos infantis (e.g., memória, dominó, pula pirata, quebra cabeça) foram utilizados para as atividades realizadas após cada sessão.

Para a programação das tarefas experimentais e registro das respostas foi utilizado o software Contingência Programada (desenvolvido por Luiz A. V. Batitucci, Jassanã S. L. Batitucci e Elenice S. Hanna, 2007), para o 
sistema Windows. Os estímulos eram apresentados em um microcomputador HP Pavilion dv5-2000, acoplado a um monitor de 15 polegadas da marca Elo, com tela sensível ao toque, e um headphone Multilaser, destinado à criança, para auxiliar a mascarar sons externos.

\section{Estímulos}

Foram utilizados 33 estímulos visuais, coloridos, com 4 $\mathrm{cm}^{2}$, organizados em 11 conjuntos com três elementos cada. Três conjuntos eram compostos por figuras familiares (e.g., triângulo, estrela, lápis) e oito conjuntos eram formados por figuras não representacionais, sem referentes na língua portuguesa, selecionados da biblioteca do software Match to Sample Program (MTS), desenvolvido por William Dube e Eric Hiris (Dube, 1991).

\section{Procedimento}

Para verificar o efeito de tentativas de revisão das relações condicionais treinadas (linha de base) antes dos testes de formação e reorganização de classes, cada dupla de crianças foi exposta a duas condições experimentais, em que eram utilizados quatro conjuntos de estímulos diferentes em cada condição. Entre as condições experimentais foi manipulada a inclusão de tentativas de revisão de linha de base antes dos testes. Na Condição 1, os testes foram precedidos por tentativas de revisão de linha de base, enquanto, na Condição 2, as revisões de linha de base foram omitidas. Foram utilizados estímulos diferentes em cada condição experimental. Os participantes $\mathrm{P} 2$ e P4 foram expostos à Condição $1 \mathrm{e}$, posteriormente, à Condição 2 e para os participantes P5 e P6 a ordem de exposição às condições foi invertida.

Procedimento geral. Inicialmente, todos os participantes foram expostos à Tarefa Preliminar. Posteriormente, cada dupla foi exposta a uma das duas condições experimentais. Cada condição experimental era composta por três fases. Na Fase 1 (Estabelecimento da Linha de Base Original), foram treinadas três discriminações condicionais (AC, BC e AD) e verificada a formação de três classes de estímulos de equivalência com quatro elementos cada. $\mathrm{Na}$ Fase 2 (Reversão $\mathrm{AD}$ e Testes de Reorganização de Classes), a linha de base original foi modificada a partir do treino com reversão da relação $\mathrm{AD}$, sendo mantido o treino das relações AC e BC, e a seguir foi testada a reorganização das classes (testes de Reflexividade, Simetria e Transitividade), conforme sequência apresentada na Tabela 1.

Nas duas condições experimentais, foi utilizado o procedimento de pareamento ao modelo visual-visual nos treinos e testes de relações condicionais. Em todas as tentativas de treino, inicialmente era apresentado o estímulo modelo e a criança era solicitada a tocar na figura (estímulo modelo). A resposta de tocar no estímulo modelo resultava na apresentação dos estímulos de comparação e a criança era solicitada a selecionar a figura que correspondia ao estímulo modelo. Respostas corretas resultavam na apresentação das seguintes consequências: (a) remoção de todos os estímulos da tela; (b) apresentação, por 1,5 s, de uma tela cinza com um desenho de tema infantil, concomitante com a apresentação de sons (e.g., aplausos, "parabéns", "muito bem”), e reforço social por parte do experimentador; e (c) apresentação de uma tela cinza por 1,5 s (Intervalo entre Tentativas - ITI) que era seguido por uma nova tentativa. Respostas incorretas resultavam na apresentação de uma tela azul por $1,5 \mathrm{~s}$, seguida pelo ITI de 1,5 s e, posteriormente, era apresentada uma nova tentativa.

Nas tarefas de teste não eram apresentadas consequências diferenciais para respostas corretas ou incorretas. Todas as respostas de seleção do estímulo de comparação correspondente ao modelo resultavam na apresentação da tela cinza por $3 \mathrm{~s}$ (ITI), seguido da apresentação de uma nova tentativa.

A coleta de dados teve a duração de aproximadamente 11 semanas. Em média, foram realizadas quatro sessões por semana com cada criança, com duração de 10 a 20 min. Ao final de cada sessão, a criança brincava por $5 \mathrm{~min}$ com um brinquedo, ou jogo, selecionado previamente pelo experimentador (e.g., memória, tapa-certo, pula pirata).

Tarefa Preliminar. Foram ensinadas as habilidades motoras necessárias para utilizar a tela sensível ao toque, os desempenhos envolvidos nas tarefas de discriminação condicional de treino e teste, e as consequências para respostas corretas e incorretas. Adicionalmente, foi verificada a formação de classes de equivalência a partir de treinos de discriminações condicionais com estímulos familiares, que foi utilizado como critério para iniciar a primeira condição experimental.

A Tarefa Preliminar era composta por três sessões. Na primeira sessão, foi realizado o treino de pareamento arbitrário entre os estímulos dos conjuntos $\mathrm{X}$ (retângulo, círculo e triângulo) e Y (estrela, coração e raio) e, na segunda sessão, o treino entre os estímulos dos conjuntos $\mathrm{Y}$ (estrela, coração e raio) e Z (lápis, chupeta e cadeira). $\mathrm{Na}$ terceira sessão, as seis relações condicionais previamente ensinadas eram apresentadas de maneira alternada em três blocos mistos e era exigido o critério de $100 \%$ de acerto no último bloco. Posteriormente, era realizado o teste de equivalência ZX, o qual era composto por nove tentativas, três para cada uma das três discriminações condicionais (Z1X1, Z2X2, Z3X3). Em caso de erros nas tentativas do teste de equivalência, era repetido o treino misto na sessão seguinte, e desempenhos precisos resultavam na exposição à Fase 1 da primeira condição experimental definida para cada dupla de participantes, conforme Tabela 2, na seção Resultados.

Fase 1 - Estabelecimento da Linha de Base Original. A Fase 1 era composta por treinos das relações condicionais $\mathrm{AC}, \mathrm{BC}$ e $\mathrm{AD}$, em sessões distintas, treinos mistos e testes de formação de classes de equivalência. 
Tabela 1

Sequência de treinos e testes realizados nas fases 1, 2 e 3 da Condição 1(Com Revisão) e da Condição 2 (Sem Revisão) e o número mínimo de tentativas de cada relação.

\begin{tabular}{|c|c|c|}
\hline Condição 1 & & Condição 2 \\
\hline Tipos de tentativas & $\mathrm{N}^{\mathrm{o}}$ mínimo de tent. & Tipos de tentativas \\
\hline \multicolumn{3}{|c|}{ Fase 1 - Estabelecimento da Linha de Base Original } \\
\hline $\mathrm{AC}$ & 51 & $\mathrm{AC}$ \\
\hline $\mathrm{BC}$ & 51 & $\mathrm{BC}$ \\
\hline Treino Misto AC,BC & 18 & Treino Misto AC,BC \\
\hline $\mathrm{AD}$ & 51 & $\mathrm{AD}$ \\
\hline Treino Misto CRF & 27 & Treino Misto CRF \\
\hline Treino Misto VR2 & 18 & Treino Misto VR2 \\
\hline Revisão de $\mathrm{LB}(\mathrm{AC}, \mathrm{BC}, \mathrm{AD})$ & 18 & \\
\hline $\mathrm{AC}, \mathrm{BC}, \mathrm{AD}(\underline{\mathrm{AA}}, \mathrm{BB}, \mathrm{CC}, \mathrm{DD})$ & $9(12)$ & $\mathrm{AC}, \mathrm{BC}, \mathrm{AD}(\underline{\mathrm{AA}, \mathrm{BB}, \mathrm{CC}, \mathrm{DD}})$ \\
\hline Revisão de LB (AC,BC,AD) & 18 & \\
\hline $\mathrm{AB}, \mathrm{BC}, \mathrm{AD}(\underline{\mathrm{CA}, \mathrm{CB}, \mathrm{DA}})$ & $9(9)$ & $\mathrm{AB}, \mathrm{BC}, \mathrm{AD}(\underline{\mathrm{CA}, \mathrm{CB}, \mathrm{DA}})$ \\
\hline Revisão de LB (AC,BC,AD) & 18 & \\
\hline $\mathrm{AC}, \mathrm{BC}, \mathrm{AD}(\underline{\mathrm{AB}, \mathrm{BA}, \mathrm{BD}, \mathrm{DB}})$ & $9(12)$ & $\mathrm{AC}, \mathrm{BC}, \mathrm{AD}(\underline{\mathrm{AB}, \mathrm{BA}, \mathrm{BD}, \mathrm{DB}})$ \\
\hline \multicolumn{3}{|c|}{ Fase 2 - Reverção AD e Testes de Reorganização de classes } \\
\hline Treino Misto ADr CRF & 36 & Treino Misto ADr CRF \\
\hline Treino Misto ADr VR2 & 18 & Treino Misto ADr VR2 \\
\hline Revisão de LB Revertida (AC,BC,ADr) & 18 & \\
\hline $\mathrm{AC}, \mathrm{BC}, \mathrm{ADr}(\underline{\mathrm{AA}, \mathrm{BB}, \mathrm{CC}, \mathrm{DD}})$ & $9(12)$ & $\mathrm{AC}, \mathrm{BC}, \mathrm{ADr}(\underline{\mathrm{AA}, \mathrm{BB}, \mathrm{CC}, \mathrm{DD}})$ \\
\hline Revisão de LB Revertida (AC,BC,ADr) & 18 & \\
\hline $\mathrm{AC}, \mathrm{BC}, \mathrm{ADr}(\underline{\mathrm{CA}, \mathrm{CB}, \mathrm{DA}})$ & $9(9)$ & $\mathrm{AC}, \mathrm{BC}, \mathrm{ADr}(\underline{\mathrm{CA}, \mathrm{CB}, \mathrm{DA}})$ \\
\hline Revisão de LB Revertida (AC,BC,ADr) & 18 & \\
\hline $\mathrm{AC}, \mathrm{BC}, \mathrm{ADr}(\underline{\mathrm{AB}, \mathrm{BA}, \mathrm{BD}, \mathrm{DB}, \mathrm{CD}, \mathrm{DC}})$ & $9(18)$ & $\mathrm{AC}, \mathrm{BC}, \mathrm{ADr}(\underline{\mathrm{AB}, \mathrm{BA}, \mathrm{BD}, \mathrm{DB}, \mathrm{CD}, \mathrm{DC}})$ \\
\hline \multicolumn{3}{|c|}{ Fase 3 - Restabelecimento da Linha de Base Original } \\
\hline Treino Misto CRF & 36 & Treino Misto CRF \\
\hline Treino Misto VR2 & 18 & Treino Misto VR2 \\
\hline Revisão de LB (AC,BC,AD) & 18 & \\
\hline $\mathrm{AC}, \mathrm{BD}, \mathrm{AD}(\underline{\mathrm{AA}, \mathrm{BB}, \mathrm{CC}, \mathrm{DD}})$ & $9(12)$ & $\mathrm{AC}, \mathrm{BD}, \mathrm{AD}(\underline{\mathrm{AA}, \mathrm{BB}, \mathrm{CC}, \mathrm{DD}})$ \\
\hline Revisão de LB (AC,BC,AD) & 18 & \\
\hline $\mathrm{AC}, \mathrm{BD}, \mathrm{AD}(\underline{\mathrm{CA}}, \mathrm{CB}, \mathrm{DA})$ & $9(9)$ & $\mathrm{AC}, \mathrm{BD}, \mathrm{AD}(\underline{\mathrm{CA}}, \mathrm{CB}, \mathrm{DA})$ \\
\hline Revisão de LB (AC,BC,AD) & 18 & \\
\hline $\mathrm{AC}, \mathrm{BD}, \mathrm{AD}(\underline{\mathrm{AB}, \mathrm{BA}, \mathrm{BD}, \mathrm{DB}, \mathrm{CD}, \mathrm{DC})})$ & $9(18)$ & $\mathrm{AC}, \mathrm{BD}, \mathrm{AD}(\underline{\mathrm{AB}, \mathrm{BA}, \mathrm{BD}, \mathrm{DB}, \mathrm{CD}, \mathrm{DC})}$ \\
\hline
\end{tabular}

Nota: Os itens sublinhados indicam as relações testadas

Treino das relações condicionais. Todas as sessões de treino (AC, $\mathrm{BC}$ e $\mathrm{AD})$ eram compostas por 51 tentativas, organizadas em 14 blocos que continham de uma a seis tentativas. As três relações condicionais de cada treino eram ensinadas, uma por vez (e.g., $\mathrm{A} 1 \mathrm{C} 1, \mathrm{~A} 2 \mathrm{C} 2$ e, por último, $\mathrm{A} 3 \mathrm{C} 3$, com 3,4 e 5 blocos, respectivamente). Como procedimento para reduzir a quantidade de erros, nesses blocos de treino de cada relação condicional, na tentativa inicial era apresentada apenas um estímulo de comparação $(\mathrm{S}+)$ e, ao longo das demais tentativas, o número de comparações aumentava gradualmente até três. Os dois últimos blocos continham seis tentativas e eram apresentadas tentativas misturadas das três relações. Para avançar de um bloco a outro, era necessário atingir o critério de $100 \%$ de acerto. Em caso de erro, o bloco era repetido por, no máximo, três vezes e, se na terceira exposição o critério não fosse atingido, a sessão era encerrada e o treino era repetido na próxima sessão. O critério de finalização do treino era $100 \%$ de acerto no bloco final. 
Após o treino das relações $\mathrm{AC}$ e $\mathrm{BC}$, foi realizado o treino misto $\mathrm{AC} / \mathrm{BC}$ em que eram apresentados três blocos com seis tentativas, sendo uma tentativa de cada relação condicional (A1C1, A2C2, A3C3, B1C1, B2C3, B3C3). Em seguida, foi realizado o treino da relação $\mathrm{AD}$.

O Treino Misto CRF, realizado após o Treino AD, era composto por 27 tentativas, organizadas em três blocos de nove tentativas, com uma tentativa para cada relação condicional previamente treinada $(\mathrm{A} 1 \mathrm{C} 1, \mathrm{~A} 2 \mathrm{C} 2, \mathrm{~A} 3 \mathrm{C} 3$, B1C1, B2C3, B3C3, A1D1, A2D2, A3D3) e reforçamento contínuo (CRF) programado para todas as respostas corretas. Após o treino Misto CRF foi realizado o Treino Misto VR2, em que o reforço era apresentado, em média, a cada duas respostas corretas (VR2), com o objetivo de preparar o participante para os testes, realizados em extinção. O Treino Misto VR2 era composto por dois blocos de nove tentativas e foram utilizados critérios similares aos dos treinos das relações condicionais separadas.

Testes de formação de classes de equivalência com Revisão de Linha de Base. Foram realizadas três sessões de teste (Reflexividade, Simetria e Equivalência). Na Condição 1, cada teste era composto por três blocos: (1) Treino Misto CRF (nove tentativas); (2) Treino Misto VR2 (nove tentativas); e (3) Teste de Reflexividade, Simetria ou Transitividade/Equivalência. Os dois blocos de treino misto, que precediam os testes, são considerados como revisão da linha de base, pois envolvem exposição a tentativas adicionais das relações previamente treinadas. $\mathrm{O}$ número de tentativas era diferente para cada teste.

O participante somente era exposto ao bloco de teste após atingir o critério de $100 \%$ de acerto nos dois blocos de treino misto. Os testes foram realizados em sonda, ou seja, as tentativas de teste, sem consequências diferenciais programadas para acerto e erro (extinção), eram intercaladas por tentativas das relações de linha de base, com reforçamento em CRF programado para as respostas corretas. Em média, cada duas tentativas de teste eram seguidas por uma tentativa de treino.

Na sessão de Teste de Reflexividade, o bloco de teste era composto por 21 tentativas, sendo 12 de relações de identidade (AA, BB, CC, DD) e nove de relações de linhas de base. No Teste de Simetria o bloco de teste continha 18 tentativas, nove de relações simétricas (CA, CB, DA) e nove de linha de base. Na sessão de Teste de Equivalência, o bloco de teste era formado por 21 tentativas, 12 das relações de equivalência ( $\mathrm{AB}, \mathrm{BA}, \mathrm{BD}, \mathrm{DB})$ e nove de linha de base. Em todos os testes foi apresentada apenas uma tentativa de cada relação.

Para a Fase 1 foi definido como critério de formação de classes de equivalência (Classe 1 - A1B1C1D1; Classe 2 - A2B2C2D2; Classe 3 - A3B3C3D3), para as três sessões de teste (Reflexividade, Simetria, Equivalência), desempenho no bloco de teste com $100 \%$ de acerto nas tentativas de relações de linha de base e, no mínimo, $88 \%$ de acerto (apenas um erro) nas tentativas de teste. Caso este critério não fosse atingido em um dos testes, a sessão de teste correspondente era repetida.

Testes de formação de classes de equivalência sem Revisão de Linha de Base. Conforme Tabela 1, na Condição 2 os testes de formação de classes de equivalência foram realizados de maneira similar aos testes da Condição 1, porém em cada sessão de teste os dois blocos de Treino Misto, que precediam o bloco de teste, foram omitidos.

Fase 2 - Reversão (AD) e Testes de Reorganização de Classes. A Fase 2 era formada por treinos mistos com reversão da relação $\mathrm{AD}(\mathrm{ADr})$ e testes de reorganização de classes.

Treino Misto com reversão da relação $A D$ em CRF (Treino Misto ADr CRF). Este treino era formado por tentativas de treino de reversão da relação AD (Treino ADr) e Treino Misto ADr. Na Fase 1, foram treinadas as relações condicionais A1D1, A2D2 e A3D3. No Treino ADr da Fase 2, foram ensinadas novas relações entre os elementos dos conjuntos A e D: Na presença do modelo A1, o participante deveria escolher D3 (A1D3); quando o modelo era A2, a escolha correta era D1 (A2D1); e quando o modelo era A3, a escolha de $\mathrm{D} 2$ era reforçada (A3D2).

O bloco de Treino ADr era formado por nove tentativas. As três discriminações condicionais foram treinadas uma de cada vez (A1D3, A2D1 e, por último, A3D2), sendo que o número de comparações aumentava gradualmente de um até três (o $\mathrm{S}+$ e dois $\mathrm{S}-$ ). $\mathrm{O}$ bloco de treino $\mathrm{ADr}$ era seguido por três blocos de nove tentativas cada, em que eram apresentadas tentativas misturadas das relações da linha de base original $\mathrm{AC}$ e $\mathrm{BC}$ com as tentativas da relação ADr (Treino Misto ADr). Após o Treino Misto ADr CRF, foi realizado o Treino Misto ADr VR2. O Treino Misto ADr VR2 apresentava a mesmas características do Treino Misto VR2 da Fase 2. Entretanto, as tentativas AD foram substituídas por tentativas ADr.

Foram utilizados os mesmos critérios de repetição de blocos e de finalização do treino descritos na Fase 1 para os Treinos Mistos CRF e Treino Misto VR2.

Testes de Reorganização de Classes. Foram realizados testes similares aos da Fase 1 a fim de verificar se, após a reversão da relação $\mathrm{AD}$, as classes de equivalência originais seriam mantidas ou se as classes seriam modificadas de acordo com as contingências do treino mais recente, o que poderia resultar em: Classe 1 - A1B1C1D3, Classe 2 - A2B2C2D1 e Classe 3 - A3B3C3D2. Na sessão de teste de equivalência foram inseridas seis tentativas de teste $\mathrm{DC}$ e $\mathrm{CD}$, sendo uma para cada relação. Diferentemente da Fase 1, não foi utilizado critério de formação de classes no bloco de teste de cada um dos três testes, sendo que a sessão era finalizada após o participante concluir as tentativas programadas. Conforme Tabela 1, na Condição 1 os testes foram precedidos por Revisão de Linha de Base e na Condição 2 os blocos de tentativas de Revisão de Linha de Base foram omitidos. 
Fase 3 - Restabelecimento da Linha de Base Original. Na Fase 3 foram repetidos o Treino Misto CRF, o Treino Misto VR2 e os testes da Fase 1, com a inclusão de tentativas $\mathrm{CD}$ e DC no teste de equivalência. O objetivo dessa fase foi verificar se com a mudança nas contingências de treino, treino da relação $\mathrm{AD}$ (restabelecimento da Linha de Base Original), as classes seriam novamente reorganizadas e resultariam no restabelecimento das classes formadas na Fase 1 (Classe 1 - A1B1C1D1, Classe 2 - A2B2 C2D2 e Classe 3 - A3B3C3D3).

\section{RESULTADOS}

$\mathrm{Na}$ Etapa Preliminar, com estímulos familiares, foram necessárias de quatro a seis sessões para os participantes atingirem o critério de aprendizagem dos treinos. Foi verificada a formação de classes de equivalência $(100 \%$ de acerto em nove tentativas), na primeira ou na segunda exposição ao teste de equivalência. Portanto, os quatro participantes atingiram o critério para a realização das tarefas das condições experimentais.

Para as duas condições, o desempenho em todas as tentativas de treino das relações condicionais (AC, BC e AD), apresentadas separadas ou em conjunto (treinos misto CRF e VR2), incluindo as tentativas de revisão de linha de base da Condição 1 , foi analisado nas três fases considerando o total de tentativas necessárias para atingir os critérios dos treino (Tentativas), o número de tentativas que excedia o número mínimo (Tent. Excedentes) e o total de erros (Tabela 2). Na Fase 1 a maior quantidade de erros na Condição 1 (com revisão de linha de base) para os participantes P4, P5 e P6 (9-16 erros) resultou em exposição a mais tentativas (40-71) do que na Condição 2, além do mínimo previsto (Condição 1 - 270; Condição 2- 216). Diferentemente, na Fase 2, os participantes apresentaram mais erros (5-19) na primeira condição a que foram expostos (P2 e P4 - Condição 1; P5 e P6 - Condição 2). Em função dos critérios de aprendizagem utilizados, tais erros resultaram na exposição adicional a 63-99 tentativas. A maioria dos participantes necessitaram de uma quantidade menor de tentativas para restabelecer a linha de base original (Fase 3) em comparação com a Fase 2 (exceto P6). A quantidade total de erros diminuiu para, no máximo, sete erros na Fase 3 , sendo que os maiores valores foram obtidos na Condição 1 (P4 e P6) e a quantidade de tentativas excedentes foi de 45 e 66 , respectivamente.
O desempenho mínimo de $89 \%$ de acerto foi utilizado como critério de formação (Fase 1), reorganização (Fase 2) e restabelecimento das classes originais (Fase 3). Foi considerado o valor médio para cada um dos três tipos de teste: reflexividade (AA, BB, CC e DD), simetria (DA, $\mathrm{CA}$ e $\mathrm{CB}$ ) e transitividade/equivalência (BA, $\mathrm{AB}, \mathrm{BD}, \mathrm{DB}$, CD e DC), os quais estão apresentados na Tabela 3. Nas duas condições todos os participantes formaram classes de equivalência na Fase 1. Na Condição 1 (Com Revisão) três participantes (P2, P5 e P6) reorganizaram as classes na Fase 2 e, posteriormente, restabeleceram as classes originais na Fase 3. A exceção foi P4 que atingiu o critério de reorganização de classes nos testes de reflexividade e de simetria nas fases 2 e 3, mas não nos testes de transitividade/ equivalência. Entretanto, na Condição 2 (Sem Revisão) os participantes atingiram o critério de reorganização de classes (Fase 2) apenas nos testes de reflexividade, exceto P5 que também atingiu esse critério nos testes de simetria. Três participantes (P2, P4 e P6) restabeleceram as classes originais na Fase 3 e um (P5) não atingiu o critério apenas nos testes de transitividade/equivalência, embora o valor tenha sido muito próximo.

A Tabela 4 apresenta o desempenho dos participantes em cada teste de simetria (CA, CB e DA) e de transitividade/ equivalência (BA, $\mathrm{AB}, \mathrm{BD}, \mathrm{DB}, \mathrm{CD}$ e $\mathrm{DC})$ das condições $1 \mathrm{e}$ 2, nas fases 2 e 3 . O desempenho nas três tentativas de teste da cada relação foi classificado de acordo com a coerência com a contingência da linha de base original $(\mathrm{O})$, revertida (R), ou com nenhuma das contingências de treino de linha de base $(\mathrm{N})$. Os números de 0-3 indicam a quantidade de respostas corretas de cada categoria $(\mathrm{O}, \mathrm{R}$ ou $\mathrm{N})$.

Tabela 2

Número de tentativas realizadas, número de tentativas excedentes e total de erros nos treinos $A C, B C$ e AD e treinos mistos CRF e VR2 nas três fases das condições 1 (Com Revisão) e 2 (Sem Revisão), por ordem de exposição

\begin{tabular}{lcccccccccc}
\hline \multirow{2}{*}{ Participantes } & Condição & \multicolumn{3}{c}{ Tentativas } & \multicolumn{3}{c}{ Tent. excedentes } & \multicolumn{3}{c}{ Total de erros } \\
\cline { 2 - 10 } & Fase 1 & Fase 2 & Fase 3 & Fase 1 & Fase 2 & Fase 3 & Fase 1 & Fase 2 & Fase 3 \\
\hline P2 & 1 & 274 & 171 & 108 & 4 & 63 & 0 & 1 & 5 & 0 \\
& 2 & 222 & 72 & 63 & 6 & 18 & 9 & 1 & 3 & 1 \\
P4 & 1 & 314 & 174 & 153 & 44 & 66 & 45 & 10 & 7 & 5 \\
& 2 & 237 & 90 & 57 & 21 & 36 & 3 & 4 & 5 & 1 \\
P5 & 2 & 246 & 138 & 75 & 30 & 84 & 21 & 4 & 8 & 1 \\
& 1 & 310 & 147 & 117 & 40 & 39 & 9 & 9 & 3 \\
P6 & 2 & 228 & 153 & 87 & 12 & 99 & 33 & 3 & 19 & 3 \\
& 1 & 341 & 153 & 174 & 71 & 45 & 66 & 16 & 6 & 7 \\
\hline
\end{tabular}


Tabela 3

Porcentagem média de acerto para cada teste das propriedades emergentes nas fases 1, 2 e 3 da Condição 1(Com Revisão) e da Condição 2 (Sem Revisão)

\begin{tabular}{|c|c|c|c|c|c|c|c|c|}
\hline \multirow{2}{*}{ Testes } & \multicolumn{4}{|c|}{ Condição 1 (Com Revisão) } & \multicolumn{4}{|c|}{ Condição 2 (Sem Revisão) } \\
\hline & P2 & P4 & P5 & P6 & P2 & P4 & P5 & P6 \\
\hline \multicolumn{9}{|l|}{ Fase 1} \\
\hline Reflexividade & 100 & 100 & 100 & 100 & 100 & 100 & 100 & 100 \\
\hline Simetria & 89 & 100 & 89 & 100 & 89 & 89 & 89 & 89 \\
\hline Trans./Equiv. & 100 & 100 & 100 & 100 & 100 & 100 & 100 & 100 \\
\hline \multicolumn{9}{|l|}{ Fase 2} \\
\hline Reflexividade & 100 & 100 & 100 & 100 & 100 & 100 & 100 & 100 \\
\hline Simetria & 89 & 100 & 89 & 100 & 78 & 78 & 89 & 67 \\
\hline Trans./Equiv. & 100 & 67 & 100 & 89 & 78 & 61 & 61 & 83 \\
\hline \multicolumn{9}{|l|}{ Fase 3} \\
\hline Reflexividade & 100 & 100 & 100 & 100 & 100 & 100 & 100 & 100 \\
\hline Simetria & 100 & 100 & 89 & 100 & 89 & 100 & 100 & 89 \\
\hline Trans./Equiv. & 100 & 83 & 94 & 100 & 94 & 100 & 83 & 100 \\
\hline
\end{tabular}

Nota. Dados sombreados de cinza indicam desempenhos abaixo do critério de $89 \%$ de acerto.

Tabela 4

Desempenho nos testes de simetria e transitividadelequivalência nas fases 2 e 3 da Condição 1(Com Revisão) e da Condição 2 (Sem Revisão)

\begin{tabular}{|c|c|c|c|c|c|c|c|c|c|c|c|c|c|c|c|c|c|c|c|c|c|c|c|c|}
\hline \multicolumn{13}{|c|}{ Condição 1 (Com Revisão) } & \multicolumn{12}{|c|}{ Condição 2 (Sem Revisão) } \\
\hline \multirow[t]{2}{*}{ Testes } & \multicolumn{3}{|c|}{$\mathrm{P} 2$} & \multicolumn{3}{|c|}{$\mathrm{P} 4$} & \multicolumn{3}{|c|}{ P5 } & \multicolumn{3}{|c|}{ P6 } & \multicolumn{3}{|c|}{$\mathrm{P} 2$} & \multicolumn{3}{|c|}{ P4 } & \multicolumn{3}{|c|}{ P5 } & \multicolumn{3}{|c|}{ P6 } \\
\hline & $\mathrm{O}$ & $\mathrm{R}$ & $\mathrm{N}$ & $\mathrm{O}$ & $\mathrm{R}$ & $\mathrm{N}$ & $\mathrm{O}$ & $\mathrm{R}$ & $\mathrm{N}$ & $\mathrm{O}$ & $\mathrm{R}$ & $\mathrm{N}$ & $\mathrm{O}$ & $\mathrm{R}$ & $\mathrm{N}$ & $\mathrm{O}$ & $\mathrm{R}$ & $\mathrm{N}$ & $\mathrm{O}$ & $\mathrm{R}$ & $\mathrm{N}$ & $\mathrm{O}$ & $\mathrm{R}$ & $\mathrm{N}$ \\
\hline \multicolumn{25}{|c|}{ Fase 2 - Reversão AD e Testes de Reorganização de Classes } \\
\hline $\mathrm{CA}$ & 3 & 0 & 0 & 2 & $\mathbf{0}$ & 1 & 3 & 0 & 0 & 3 & 0 & 0 & 3 & 0 & 0 & 3 & 0 & 0 & 3 & $\mathbf{0}$ & 0 & 2 & 1 & 0 \\
\hline $\mathrm{CB}$ & 3 & 0 & $\mathbf{0}$ & 2 & 1 & 0 & 3 & 0 & 0 & 3 & 0 & 0 & 3 & 0 & 0 & 3 & 0 & 0 & 3 & 0 & 0 & 2 & 0 & 1 \\
\hline BA & 3 & $\mathbf{0}$ & 0 & 3 & $\mathbf{0}$ & 0 & 3 & 0 & 0 & 3 & 0 & 0 & 3 & 0 & 0 & 3 & 0 & 0 & 3 & 0 & 0 & 3 & 0 & 0 \\
\hline $\mathrm{AB}$ & 3 & $\mathbf{0}$ & 0 & 3 & $\mathbf{0}$ & 0 & 3 & 0 & 0 & 3 & 0 & 0 & 3 & 0 & 0 & 3 & 0 & 0 & 2 & 1 & 0 & 3 & 0 & 0 \\
\hline $\mathrm{DB}^{\mathrm{a}}$ & 0 & 3 & $\mathbf{0}$ & 2 & 1 & $\mathbf{0}$ & 0 & 3 & 0 & 1 & 2 & 0 & 0 & 3 & 0 & 2 & 1 & 0 & 1 & 2 & 0 & 3 & 0 & 0 \\
\hline $\mathrm{DA}^{\mathrm{a}}$ & 1 & 2 & 0 & 2 & 1 & $\mathbf{0}$ & 0 & 3 & 0 & 0 & 3 & 0 & 2 & 1 & 0 & 2 & 1 & 0 & 1 & 2 & 0 & 1 & 2 & 0 \\
\hline $\mathrm{BD}^{\mathrm{a}}$ & 0 & 3 & 0 & 1 & 2 & 0 & 0 & 3 & 0 & 0 & 3 & 0 & 1 & 2 & 0 & 1 & 2 & 0 & 2 & 1 & 0 & 1 & 2 & 0 \\
\hline $\mathrm{CD}^{\mathrm{a}}$ & 0 & 3 & 0 & 0 & 3 & $\mathbf{0}$ & 1 & 2 & 0 & 0 & 3 & 0 & 1 & 2 & 0 & 0 & 2 & 1 & 1 & 2 & 0 & 0 & 3 & 0 \\
\hline $\mathrm{DC}^{\mathrm{a}}$ & $\mathbf{0}$ & 3 & 0 & 3 & $\mathbf{0}$ & $\mathbf{0}$ & 0 & 3 & 0 & 1 & 2 & 0 & 1 & 2 & 0 & 0 & 2 & 1 & 2 & 1 & 0 & 2 & 1 & 0 \\
\hline \multicolumn{25}{|c|}{ Fase 3 - Restabelecimento da Linha de Base Original } \\
\hline $\mathrm{CA}$ & 3 & 0 & 0 & 3 & 0 & 0 & 3 & 0 & 0 & 3 & 0 & 0 & 3 & 0 & 0 & 3 & 0 & 0 & 3 & 0 & 0 & 2 & 1 & 0 \\
\hline CB & 3 & $\mathbf{0}$ & 0 & 3 & $\mathbf{0}$ & 0 & 3 & 0 & 0 & 3 & 0 & 0 & 3 & 0 & 0 & 3 & 0 & 0 & 3 & 0 & 0 & 3 & 0 & $\mathbf{0}$ \\
\hline BA & 3 & 0 & o & 3 & 0 & 0 & 3 & 0 & 0 & 3 & 0 & 0 & 3 & 0 & 0 & 3 & 0 & 0 & 3 & 0 & 0 & 3 & 0 & 0 \\
\hline $\mathrm{AB}$ & 3 & 0 & 0 & 3 & 0 & $\mathbf{0}$ & 3 & 0 & 0 & 3 & 0 & 0 & 3 & 0 & 0 & 3 & 0 & 0 & 3 & 0 & 0 & 3 & $\mathbf{0}$ & 0 \\
\hline DB & 3 & 0 & $\mathbf{0}$ & 3 & 0 & 0 & 3 & 0 & 0 & 3 & 0 & 0 & 3 & 0 & 0 & 2 & 1 & 0 & 2 & 0 & 1 & 3 & 0 & $\mathbf{0}$ \\
\hline DA & 3 & 0 & $\mathbf{0}$ & 3 & 0 & 0 & 3 & 0 & 0 & 3 & 0 & 0 & 2 & 0 & 1 & 3 & 0 & 0 & 3 & 0 & $\mathbf{0}$ & 3 & 0 & $\mathbf{0}$ \\
\hline $\mathrm{BD}$ & 3 & $\mathbf{0}$ & 0 & 3 & 0 & $\mathbf{0}$ & 3 & 0 & 0 & 3 & 0 & 0 & 3 & 0 & 0 & 3 & 0 & 0 & 3 & $\mathbf{0}$ & 0 & 3 & 0 & 0 \\
\hline $\mathrm{CD}$ & 3 & 0 & 0 & 2 & 1 & 0 & 1 & 2 & 0 & 3 & 0 & 0 & 3 & 0 & 0 & 2 & 0 & 1 & 2 & 0 & 1 & 3 & 0 & 0 \\
\hline DC & 3 & 0 & 0 & 3 & 0 & 0 & 3 & 0 & 0 & 3 & 0 & 0 & 2 & 1 & 0 & 2 & 1 & 0 & 2 & 0 & 1 & 3 & 0 & 0 \\
\hline
\end{tabular}

Nota. O - respostas coerentes com a linha de base original; $\mathrm{R}$ - respostas coerentes com a linha de base revertida; $\mathrm{N}$ - respostas coerentes com nenhuma das contingências de treino; dados sombreados de cinza indicam a primeira condição realizada.

${ }^{a}$ Relações nas quais as respostas deveriam ser coerentes com a linha de base revertida (R). 
Após a reversão da relação $\mathrm{AD}$ (Fase 2), verifica-se nas duas condições que, nas tentativas de teste das quatro relações que não envolviam os estímulos do Conjunto $\mathrm{D}(\mathrm{CA}, \mathrm{CB}$, $\mathrm{BA}$ e $\mathrm{AB}$ ), a maioria das respostas ( 3 ou 2 acertos nas três tentativas de cada relação) foram coerentes com a linha de base original $(\mathrm{O})$, sendo que na segunda condição a que foram expostos (P2 e P4 - Condição 2; P5 e P6 - Condição 1), o desempenho dos participantes foi mais preciso. Com o restabelecimento da linha de base original (Fase 3), verifica-se que o desempenho nessas quatro relações se manteve coerente com a linha de base original $(\mathrm{O})$ e apenas P6 apresentou uma resposta coerente com a linha de base revertida $(\mathrm{R})$ nas tentativas de teste da relação $\mathrm{CA}$.

Para as cinco relações testadas em que era esperado desempenho coerente com o treino de reversão (ADr) da
Fase 2 (DB, DA, BD, CD, DC) verifica-se que, nas duas condições os participantes apresentaram, para a maioria das relações testadas, mais respostas ( 3 ou 2) coerentes com a linha de base revertida (R), sendo que os desempenhos foram mais precisos na Condição 1, exceto para P4. Na Fase 3, os desempenhos na Condição 1 voltaram a ser coerentes com a linha de base original $(\mathrm{O})$, exceto para P5 (Relação $\mathrm{CD}$ ). $\mathrm{Na}$ Condição 2 resultados similares foram observados, porém com menor precisão. P2 e P4 apresentaram uma resposta coerente com a linha de base revertida $(\mathrm{R})$ para uma ou duas relações, respectivamente, e respostas não coerentes com as duas linhas de base $(\mathrm{N})$ ocorreram para uma (P2 e P4) ou três relações testadas (P6). Nas duas fases da Condição 2 a relação com mais erros foi DC.

\section{DISCUSSÃO}

O presente estudo utilizou um procedimento baseado no paradigma de equivalência de estímulos (Sidman \& Tailby, 1982), mais especificamente na literatura sobre reorganização de classes de equivalência (Pilgrim \& Galizio, 1990, 1995; Pilgrim et al., 1995; Garotti \& de Rose, 2007). Foi replicado o estudo de Garotti e de Rose (2007), com estudantes universitários, e os resultados em relação ao desempenho nos testes de reorganização e restabelecimento das classes de equivalência foram estendidos para crianças de 5 a 6 anos de idade. Nos testes da Condição 1 (Com Revisão), três participantes (P2, P5 e P6) reorganizaram as classes de equivalência após o treino de reversão $\mathrm{AD}$ $\mathrm{e}$, posteriormente, restabeleceram as classes originais. Porém, na Condição 2 (Sem Revisão), nenhum participante reorganizou as classes.

A reorganização de classes de equivalência foi observada em vários estudos anteriores (e.g., Dube et al., 1987; Folsta \& de Rose, 2007; Garotti \& de Rose, 2007; Garotti, de Souza, de Rose, Molina, \& Gil, 2000; Saunders et al., 1999). O desempenho dos participantes na Condição 1 replica, portanto, os resultados de tais estudos com crianças e com estímulos visuais não familiares. Contudo, na literatura sobre reorganização de classes é relatada a dificuldade de crianças para reorganizar as classes de equivalência (Goyos, 2000; Pilgrim et al., 1995). O desempenho dos participantes na Condição 2 (Sem Revisão) corrobora estas observações uma vez que desempenho igual ou superior a $89 \%$ de acerto foi verificado apenas para as relações de reflexividade (pareamento de identidade), o que sugere a relevância da exposição à uma maior quantidade de treino das relações de linha de base para a emergência das relações arbitrárias.

Os estudos de Pilgrim e Galizio (1990, 1995) e Pilgrim et al. (1995) descrevem uma dissociação entre os resultados nos testes de simetria e de transitividade/equivalência. Nestes estudos, apenas as relações simétricas foram coerentes com a linha de base revertida. No presente estudo, foi possível observar tal dissociação nos desempenhos nos testes das relações de simetria e de transitividade/equivalência para os quatro participantes na Condição 2 e para um participante na Condição 1 (P4) que não reorganizaram as classes. Uma possível explicação de Garotti e de Rose (2007) é que as relações simétricas envolvem a interação entre dois estímulos, e as transitivas e de equivalência envolvem três ou mais estímulos. Assim, em uma situação de contingência conflitante, quanto maior o número de estímulos envolvidos na relação, menor a probabilidade do participante apresentar desempenhos precisos. Esta afirmativa é corroborada pelo estudo de Folsta e de Rose (2007) que investigou o efeito do tamanho da classe na reorganização e foi verificado que quanto maior a classe, maior a dificuldade em reorganizá-la.

Quanto à influência da história experimental, ou seja, o efeito da ordem de exposição às duas condições experimentais, com conjuntos de estímulos diferentes, foi possível observar a formação de learning set. Este fenômeno é evidenciado quando há transferência de habilidades aprendidas para situações novas, após uma história de treino com contingências similares, o que facilita novas aprendizagens (Harlow, 1949). Ao comparar os desempenhos nos treinos (Tabela 2) de P2, P4, P5 e P6 nas duas condições experimentais, foi verificado que, na segunda condição experimental a que foram expostos, todos os participantes necessitaram de menos tentativas para atingir o critério de aprendizagem nos treinos da Fase 2, que envolvia a reversão de uma das relações de linha de base (AD). Adicionalmente, nas duas condições os participantes apresentaram menos erros na Fase 3 (restabelecimento da linha de base original) em comparação com a Fase 1.

Quanto aos testes, vale destacar uma tendência clara no desempenho dos participantes P5 e P6 nas fases 2 e 3, que apresentaram desempenhos mais precisos na Condição 1 do que na Condição 2, conforme Tabela 4. Esse resultado sugere que a exposição à tentativas de revisão de linha 
de base após a exposição à Condição 2 (Sem Revisão) auxiliou na reorganização e restabelecimento das classes. Porém, o participante P2 apresentou desempenho similar ao de P5 e P6, independentemente da ordem de exposição. Uma replicação do estudo com mais participantes sendo expostos as duas condições experimentais em diferentes ordens, ou a apenas uma condição experimental por duas vezes consecutivas (delineamentos $\mathrm{AB}, \mathrm{BA}, \mathrm{AA}$ e $\mathrm{BB}$ ), possibilitaria uma avaliação mais controlada da variável ordem de exposição.

O presente estudo consistiu em uma replicação do estudo de Garotti e de Rose (2007), mas foram efetuadas adaptações para adequar os procedimentos à faixa etária dos participantes. Uma dessas alterações consistiu na redução de uma das relações treinadas e de uma das relações revertidas no estudo original. Tal alteração está coerente com os resultados de Folsta e de Rose (2007) que verificaram que o aumento na quantidade de relações treinadas dificulta a reorganização das classes de equivalência e com o estudo de Almeida e Haydu (2009) que demonstrou que o número de relações revertidas não afetou a reorganização das classes. Adicionalmente, no estudo de Garotti e de Rose (2007) ocorreu reorganização após somente um treino de reversão e não houve diferença após o treino de uma nova relação e da reversão de uma segunda relação. Assim, a diminuição do número de fases experimentais de cinco (estudo original) para três (presente estudo) favoreceu a realização do estudo com crianças e os resultados foram coerentes com os obtidos por Garotti e de Rose (2007).

Outra diferença metodológica é que, no presente estudo, o primeiro bloco de tentativas de revisão das relações de linha de base foi realizado em CRF e o segundo bloco em VR2. Nos dois experimentos relatados por Garotti e de Rose (2007), as tentativas de revisão de linha de base eram realizadas em extinção e não foram caracterizadas como tentativas de treino. Mesmo em extinção, as tentativas de revisão de linha de base podem ter adquirido a função de fortalecer as relações de linha de base em virtude de possíveis consequências reforçadoras, relacionadas com a mudança para outro tipo de bloco de tentativas (testes) ou o término da sessão, que caracterizaria o progresso para a próxima sessão prevista no experimento. Assim, as tentativas de revisão de linhas de base, tanto no presente estudo como no de Garotti e de Rose (2007) podem ter funcionado como um treino complementar, ou um treino adicional, o que pode ter aumentado a probabilidade de desempenhos mais precisos nos testes posteriores. Investigações futuras, portanto, poderiam avaliar o efeito de testes realizados em sonda ou em blocos de tentativas de teste de estabelecimento e de reorganização de classes de equivalência.

Uma possível variável envolvida no efeito das tentativas de revisão de linha de base na reorganização de classes pode ser a proximidade temporal das tentativas de treino com as de teste. Os blocos com tentativas de revisão de linha de base eram realizados na mesma sessão que os testes. Garotti e de Rose (2007) consideraram que a variável crítica para a reorganização das classes de equivalência não está na recência do treino. Os pesquisadores argumentam que nos estudos de Pilgrim e Galizio (1990; 1995), a linha de base mais recente (original ou revertida) afetou o desempenho nos testes de simetria, mas não nos testes de transitividade/ equivalência. Sendo assim, Garotti e de Rose (2007) concluíram que as revisões de linha de base apresentam propriedade discriminativa de contexto. Porém, em Garotti e de Rose (2007), no presente estudo, e em outros estudos (e.g., Dube et al., 1987; Saunders et al., 1999) que não utilizaram revisão de linha de base, foram utilizados testes em sonda, tentativas de teste misturadas às tentativas de treino em um mesmo bloco. Nestes estudos foi possível observar que a maioria dos participantes reorganizou as classes de equivalência na Condição 2. Estes resultados podem indicar que a proximidade temporal entre as tentativas de treino com as tentativas de teste pode facilitar o maior controle das últimas relações treinadas em uma situação de contingências conflitantes, consequentemente, auxiliando na reorganização das classes. Novos estudos poderiam ser realizados para verificar o efeito das tentativas de treino temporalmente próximas às tentativas de teste na reorganização de classes.

O presente estudo acrescentou evidências em relação ao efeito da exposição às tentativas de treino de relações condicionais (revisão de linha de base) antes dos testes na formação e reorganização de classes de equivalência, na reorganização das classes. Entretanto, os resultados obtidos são inconclusivos em relação ao efeito da ordem de exposição as condições experimentais, do tipo de teste (em sonda ou em blocos) e da proximidade temporal entre as tentativas de revisão de linha de base e de teste. Adicionalmente, ainda deve ser investigada a generalidade dos resultados obtidos para outros estímulos, tais como os envolvidos em classes de equivalência culturalmente estabelecidas (e.g., racismo, obesidade, religião), e com outros procedimentos de reversão de relações condicionais. 


\section{REFERÊNCIAS}

Albuquerque, A. R., \& Melo, R. M. (2005). Equivalência de estímulos: Conceito, implicações e possibilidade de aplicação. Em J. Abreu-Rodrigues \& M. R. Ribeiro (Eds.), Análise do comportamento: Pesquisa, teoria e aplicação (pp. 245-264). Porto Alegre: ARTMED.

Almeida, J. H., \& Haydu, V. B. (2009). Reorganização de classes de estímulos equivalentes: Análise do número de estímulos de comparação. Revista Brasileira de Análise do Comportamento, 5(2), 37-50.

Barnes, D., Lawlor, H., Smeets, P. M., \& Roche, B. (1996). Stimulus equivalence and academic self-concept among mildly mentally handicapped and nonhandicapped children. The Psychological Record, 46, 87-107.

Carvalho, M. P., \& de Rose, J. C. (2014). Understanding racial attitudes through the stimulus equivalence paradigm. The Psychological Record, 64, 527-536.

de Rose, J. C., de Souza, D. G., \& Hanna, E. S. (1996). Teaching reading and spelling: Exclusion and stimulus equivalence. Journal of Applied Behavior Analysis, 29, 451-469.

Dube, W. V. (1991). Computer software for stimulus control research with Macintosh computers. Experimental Analysis of Human Behavior Bulletin, 9, 28-30.

Dube, W. V., Mcllvane, W. J., Mackay, H. A., \& Stoddard, L. T. (1987). Stimulus class membership established via stimulusreinforcer relations. Journal of the Experimental Analysis of Behavior, 47, 159-175

Dymond, S., \& Barnes, D. (1994). A transfer of self-discrimination response functions through equivalence relations. Journal of the Experimental Analysis of Behavior, 62, 251-267.

Folsta, A. G., \& de Rose, J. C. (2007). Rearrangement of equivalence classes after reversal of a single baseline relation: Influence of class size. Experimental Analysis of Human Behavior Bulletin, $25,1-5$

Garotti, M., \& de Rose, J. C. (2007). Reorganization of equivalence classes: Evidence of contextual control by baseline reviews before probes. The Psychological Record, 57, 87-102.

Garotti, M., de Souza, D. G., de Rose, J. C., Molina, R. C., \& Gil, M. S. A. (2000). Reorganization of equivalence classes after reversal of baseline relations. The Psychological Record, 40, $30-48$.

Goyos, C. (2000). Equivalence class formation via common reinforcers among prescholl children. The Psychological Record, 50, 629-654.
Hanna, E. S., Kohlsdorf, M., Quinteiro, R. S., Fava, V. M. D., de Souza, D. G., \& de Rose, J. C. (2008). Diferenças individuais na aquisição de leitura com um sistema lingüístico em miniatura. Psicologia: Teoria e Pesquisa, 24, 45-58.

Harlow, H. F. (1949). The formation of learning sets. Psychological Review, 56, 51-65.

Lynch, D. C., \& Cuvo, A. J. (1995). Stimulus equivalence instruction of fraction-decimal relations. Journal of Applied Behavior Analysis, 28, 115-126.

Pilgrim, C., Chambers, L., \& Galizio, M. (1995). Reversal of baseline relations and stimulus equivalence: II Children. Journal of the Experimental Analysis of Behavior, 63, 239-254.

Pilgrim, C., \& Galizio, M. (1990). Relations between baseline contingencies and equivalence probe performances. Journal of the Experimental Analysis of Behavior, 54, 213-224.

Pilgrim, C., \& Galizio, M. (1995). Reversal of baseline relations and stimulus equivalence: I. Adults. Journal of the Experimental Analysis of Behavior, 63, 225-238.

Prado, P. S. T., \& de Rose, J. C. (1999). Conceito de número: Uma contribuição da análise comportamental da cognição. Psicologia: Teoria e Pesquisa, 15, 227-235.

Saunders, R. R., Drake, K. M., \& Spradlin, J. E. (1999). Equivalence class establishment, expansion and modification in preschool children. Journal of the Experimental Analysis of Behavior, 71, 195-214.

Saunders, R. R., Saunders, K. J., Kirby, K. C., \& Spradlin, J. E. (1988). The merger and development of equivalence classes by unreinforced conditional selection of comparison stimuli. Journal of the Experimental Analysis of Behavior, 50, 145-162.

Sidman, M. (1994). Equivalence relations and behavior: A research story. Boston: Authors Cooperative.

Sidman, M., \& Tailby, W. (1982). Conditional discrimination vs. matching-to-sample: An expansion of the testing paradigm. Journal of the Experimental Analysis of Behavior, 8, 91-112.

Tena, R. O., \& Velázquez, H. A. (1997). Estudio exploratorio de la enseñaza de la lectura de notas musicales a través del modelo de discriminación condicional. Revista Mexicana de Psicología, 14(1), 13-29.

Watt, A., Keenan, M., Barnes, D., \& Cairns, E. (1991). Social categorization and stimulus equivalence. The Psychological Record, 41, 33-50. 